\title{
Relationship of DNA integrity to $H R G$ C633T SNP and ART outcome in infertile couples
}

\author{
Arumugam Kumaresan ${ }^{1,2}$, Anders Johannisson ${ }^{1}$, Sarah Nordqvist $^{3}$, Karin Kårehed ${ }^{3}$, \\ Helena Åkerud ${ }^{4}$, Karin E Lindgren ${ }^{3,+}$ and Jane M Morrell ${ }^{1}$ \\ ${ }^{1}$ Department of Clinical Sciences/Division of Reproduction, Swedish University of Agricultural Sciences, Uppsala, \\ Sweden, ${ }^{2}$ Theriogenology Laboratory, Animal Reproduction, Gynaecology \& Obstetrics, National Dairy Research \\ Institute, Karnal, Haryana, India, ${ }^{3}$ Department of Genetics, Immunology and Pathology, and Department of \\ Women's and Children's Health, Uppsala University, Uppsala, Sweden and ${ }^{4}$ Department of Genetics, \\ Immunology and Pathology, Uppsala University, Uppsala, Sweden
}

Correspondence should be addressed to J M Morrell; Email: jane.morrell@slu.se

${ }^{\dagger}(\mathrm{K}$ E Lindgren is now at Centre of Reproduction, Uppsala University Hospital, Uppsala, Sweden),

\begin{abstract}
The status of sperm DNA fragmentation, protamine deficiency, free thiols and disulphide bonds in colloid-selected samples and its relationship to ART outcome or HRG C633T SNP is not known. The objective of this study was to determine these relationships in spermatozoa from men with male factor or unknown factor infertility $(n=118)$ undergoing in vitro fertilisation (IVF) or intracytoplasmic sperm injection (ICSI). Sperm DNA integrity was analysed by flow cytometry using three fluorescent probes (acridine orange, monobromobimane and chromomycin A3). Principal component analysis (PCA) was used to identify the parameters that most influenced fertility. The relationships of sperm DNA integrity with seminal parameters, HRG C633T SNP and ART outcome were established using ANOVA and $t$-test. Sperm concentration and yield after preparation accounted for $27 \%$ of the total variance; sperm DNA integrity (\%DFI and disulphide bonds) accounted for $16 \%$ of the variance in men from infertile couples. Sperm $\%$ DFI was significantly higher $(\boldsymbol{P}<\mathbf{0 . 0 5})$ in older men than in younger men. A significant difference $(\boldsymbol{P}<0.01)$ was observed in \%DFI between smokers and non-smokers. Sperm \%DFI was significantly higher $(P<0.01)$ in male factor infertility compared to either female factor or unknown factor infertility while free thiols were significantly higher $(\boldsymbol{P}<0.01)$ in unknown infertility factor. No significant difference was observed between IVF success/failure in any of the seminal parameters studied. There was a tendency for protamine deficiency to be higher and disulphide concentration to be lower in men with HRG 633T. Such assessments may provide additional useful information about the prognosis for ART outcome, although more research is needed before clinical guidelines can be provided.

Reproduction (2017) 153 865-876
\end{abstract}

\section{Introduction}

Infertility is a common clinical problem affecting approximately $15 \%$ of couples around the world (de Kretser 1997, Kamath \& Bhattacharya 2012). The male factor alone is responsible for approximately $26 \%$ of these cases (Brandes et al. 2010) and contributory in another $30-40 \%$ of infertile couples (Jarow et al. 2002). As such, male factor, alone or in combination with female factor, accounts for more than $50 \%$ of cases of infertility (Esteves et al. 2012). Although the incidence of male factor infertility is increasing, it continues to remain as one of the most perplexing disorders in the reproductive field and its aetiology remains elusive (O'Flynn O'Brien et al. 2010).

Male infertility arises from a variety of problems including genetic disorders, hormonal disturbances, disorders in spermatogenesis, poor sperm quality, improper lifestyle habits and sperm DNA fragmentation (O'Flynn O'Brien et al. 2010, Esteves et al. 2012). It is estimated that more than $10 \%$ of spermatogenesis impairment is due to genetic defects (Ferlin \& Foresta 2014, Krausz et al. 2015) and there is a growing body of literature on the genetic reasons for male infertility. Interestingly, occasionally different genotypes appear to compensate for poorer sperm quality. Histidinerich glycoprotein (HRG) is an abundant plasma glycoprotein with a concentration of $100-150 \mu \mathrm{g} / \mathrm{mL}$. Its levels increase throughout life (Drasin \& Sahud 1996). Oestrogen lowers its levels in women (Hennis et al. 1995), although, to our knowledge, no studies have been done on HRG levels and male hormone levels. It is found in the testis, prostate and seminal vesicles and is 
also detected in the sperm proteome (Wang et al. 2013). Because it is a multi-domain protein, HRG is involved in forming multi-protein complexes and thus functions as an adaptor molecule (Patel et al. 2013). It is known to be involved in the regulation of angiogenesis, fibrinolysis and the immune system. HRG has been shown to enhance the clearance of apoptotic cells by acting as a bridge between naked DNA in these cells and other immunological cells and molecules (as reviewed in Blank \& Shoenfeld 2008).

The role of the HRG gene in human reproduction has been investigated recently. Although HRG C633T single nucleotide polymorphism (SNP) seems to be associated with fertility, its role in reproduction has not been studied extensively. The HRG C633 SNP, unique in humans (www.uniprot.org/uniprot/?query=histidine. rich+glycoprotein\&sort=score), codes for a proline at position 204 (Jones et al. 2005). The HRG 633T SNP codes for a serine, which allows for an additional glycolysation not found in the isoform with the proline at the same position. The roles of the HRG C633T SNP in fertility and pregnancy outcome in females have been reported (Nordqvist et al. 2011) although its role in male fertility has not been studied in detail. A recent report indicated that an SNP in the HRG gene in men was associated with IVF outcome in infertile couples; male homozygous HRG 633T SNP carriers had low sperm count, sperm concentration and motility (Lindgren et al. 2016).

In the last few decades, there has been an increasing body of research investigating the role of sperm DNA integrity in male factor infertility (Schulte et al. 2010). Growing evidence indicates that DNA damage is higher in spermatozoa of infertile men compared to that in fertile men and may have a negative effect on fertility of these patients (Evenson et al. 1999, Zini et al. 2001a, Lewis 2013). Furthermore, the estimation of sperm DNA integrity may be helpful in unexplained infertility because significant DNA fragmentation can also be present in normozoospermic sub-fertile men (Erenpreiss et al. 2008, Oleszczuk et al. 2011). While tests of sperm DNA integrity appear to have a high predictive value for outcomes of in vivo fertilisation, the relationship with the outcome of IVF and ICSI is contradictory (Schulte et al. 2010). Several researchers did not find a significant correlation between sperm DNA integrity and assisted reproduction technologies (ART) success (Morris et al. 2002, Gandini et al. 2004, Li et al. 2006) while others reported a significant reduction of pregnancy rates following IVF or ICSI among couples with high sperm DNA fragmentation (Larson et al. 2000, Benchaib et al. 2003, Virro et al. 2004, Simon et al. 2010). Evaluation of sperm DNA fragmentation is rarely incorporated in routine semen analysis in human fertility clinics (Lewis 2013, Wright et al. 2014). The current understanding of sperm
DNA damage and its effects on reproductive outcomes is far from complete and requires further investigation.

Sperm DNA fragmentation can be evaluated by a variety of tests, including comet assay, sperm chromatin structure assay (SCSA), acridine orange test, the terminal deoxynucleotidyl transferase (TdT)mediated dUTP nick end labelling (TUNEL) technique (Evenson \& Wixon 2005) or sperm chromatin dispersion test (Gosálvez et al. 2008). The TUNEL and SCSA are the most commonly used. In the recent past, there have been several advances in the techniques for evaluating sperm chromatin quality and DNA fragmentation, which includes estimation of protamine deficiency, free thiols and disulphide bonds. Protamine is a nuclear protein responsible for packing sperm chromatin, and its deficiency in spermatozoa is believed to increase the sensitivity of DNA to fragmentation. Progressive oxidation of protaminefree sulphydryl or thiol (SH) groups to disulphides in the epididymis further stabilises the compacted sperm DNA (Zini et al. 2001b). Earlier studies have shown an association between abnormal protamine deposition and infertility (Balhorn et al. 1988, Belokopytova et al. 1993), and sperm DNA integrity has also been shown to correlate with the level of free SH groups. However, the relationship among sperm DNA integrity, protamine deficiency, the level of free SH groups, conventional semen parameters and infertility has not been established.

The majority of the studies assessed sperm DNA fragmentation in unselected samples and related it with infertility or outcome of ART. However in ART, processed and selected spermatozoa are often used; density gradient centrifugation is one of the preferred techniques for sperm processing for ART. We hypothesised that assessment of the DNA integrity in selected spermatozoa rather than in unprocessed spermatozoa would be more logical to infer the ART outcome. What is not known however is the status of sperm DNA in density-gradientselected samples and how it is related to successful or unsuccessful ART outcomes. In the present retrospective study, we used flow-cytometry-based assessment of DNA fragmentation index (\%DFI), protamine deficiency, free thiols and disulphide bonds in density gradientselected spermatozoa of men from infertile couples, and related them with conventional semen parameters, HRG C633T SNP and IVF/ICSI outcome. Moreover, some lifestyle-related factors were evaluated for their influence on sperm DNA integrity and other traditional semen quality parameters.

The specific aims of the present study were: (i) to assess the relationship of HRG C633T SNP with sperm DNA integrity and other conventional semen parameters; (ii) to compare the seminal parameters and sperm DNA fragmentation status among sperm samples 
from patients with male, female and unknown factor infertility; and (iii) to study the relationship of sperm DNA integrity with ART outcome.

\section{Materials and methods}

\section{Ethics}

The study was approved by the Regional Ethics Review Board in Stockholm (diary numbers: 2007/3-31/3, 2008/689-32 and 2010/0180-32). All couples met with a healthcare professional and received written and verbal information about the study and gave written informed consent. Participation was voluntary and did not affect treatment.

\section{Study population}

Heterosexual infertile couples visiting an IVF clinic (Fertilitetscentrum, Stockholm) constituted the study population. All couples had previously undergone assessment for cause of infertility. Infertile men were screened for drug and alcohol abuse, previous infections (in testis, epididymis and prostate), previous surgery (inguinal hernia, genital tract or urinary tract) and medical history. Patients with abuse were denied treatment. Semen analyses were routinely performed by embryologists and included the concentration and motility of spermatozoa. Normally, only one semen sample was analysed during a fertility investigation, but if the results appeared to be deviant the test was repeated. Men with sperm counts below the World Health Organization guideline levels (Tocci \& Lucchini 2010) were classified as male factor infertility. Other causes were classified into either female factor or unknown depending upon the results of investigation.

In vitro fertilisation (IVF) was performed for 72 couples whereas intracytoplasmic sperm injection (ICSI) was carried out for 28 couples. The IVF treatment protocols at the Fertilitetscentrum Stockholm have previously been described in detail (Nordqvist et al. 2015). ICSI was used if the number of prepared spermatozoa using PureSperm (Nidacon, Sweden) was $\leq 1$ million or in cases with previous low fertilisation $(<50 \%)$. Embryos were assessed according to standardised clinical morphological protocols (Alpha Scientists in Reproductive Medicine \& ESHRE Special Interest Group of Embryology 2011), and transfer was not performed if there were signs of abnormal growth. The outcome of IVF or ICSI was determined by a pregnancy test on day 16 after the embryo transfer. An ultrasound was done in pregnancy weeks 6-8 and the couples subsequently reported live birth data.

\section{Sperm samples and standard semen parameter measurements}

Details of the men, such as age, medical and reproductive history, and lifestyle factors such as smoking or other forms of tobacco use (oral moist tobacco), were collected at the time of initial recruitment. Sperm samples were obtained from 119 infertile men after a recommended 2-5 days of sexual abstinence; only one ejaculate from each patient was obtained. Samples were allowed to liquefy for $30 \mathrm{~min}$. Standard semen parameters (volume, concentration and motility) were measured according to the World Health Organization guidelines (Tocci \& Lucchini 2010). For ART, ejaculate volume, sperm concentration per millilitre, total sperm concentration, progressive motility and yield after preparation (using PureSperm (Nidacon International, Sweden), a sterile colloidal silica suspension in an isotonic salt solution) were recorded for each sample. An aliquot of density gradientselected spermatozoa from each sample was stored at $-70^{\circ} \mathrm{C}$ for sperm DNA fragmentation analysis.

\section{HRG C633T SNP analysis}

A single venous blood sample was collected in EDTAcontaining tubes from each infertile man and centrifuged $(1500 \mathrm{~g}$ for $10 \mathrm{~min})$; the buffy coat was transferred to a new tube and stored at $-70^{\circ} \mathrm{C}$. Genomic DNA was extracted from the buffy coat using QIAamp DNA Blood Mini Kits (Qiagen). The samples were genotyped for the HRG C633T SNP (rs9898) using TaqMan SNP Genotyping Assay and run with StepOnePlus Real-Time PCR Systems (Life Technologies) as per the procedure described in the paper by Lindgren and coworkers (Lindgren et al. 2016).

\section{Flow cytometric analysis of sperm DNA integrity}

Sperm DNA integrity was analysed by flow cytometry using three fluorescent probes (acridine orange, monobromobimane and chromomycin A3) described below.

\section{Sperm chromatin structure assay}

Susceptibility of sperm DNA to acid-induced denaturation, an indirect method to assess DNA strand breaks, was assessed using the acridine orange (AO) assay. This assay utilises the metachromatic nucleic acid-binding dye AO, which fluoresces red or green when bound to either denatured or condensed chromatin, respectively. The sperm chromatin structure assay (SCSA) was performed as per the method given by Evenson and Jost (2000) with modifications. Briefly, spermatozoa (2 million) stored in TNE buffer $(1 \mathrm{~mL})$ were thawed on ice. $100 \mu \mathrm{L}$ sperm suspension was taken and $200 \mu \mathrm{L}$ Triton X-100 (Sigma-Aldrich) detergent solution $(0.08 \mathrm{M} \mathrm{HCL}, 0.1 \%$ Triton $\mathrm{X}-100$; $\mathrm{pH} 1.2$ ) was added. After incubation at room temperature for $30 \mathrm{~s}$, the spermatozoa were stained with $600 \mu \mathrm{L}$ of AO (SigmaAldrich) staining solution $\left(200 \mathrm{mmol} / \mathrm{L} \mathrm{Na} \mathrm{HPO}_{4}, 0.1 \mathrm{~mol} / \mathrm{L}\right.$ citric acid buffer, $\mathrm{pH} 6.0,1 \mathrm{mmol} / \mathrm{L}$ EDTA, $150 \mathrm{mmol} / \mathrm{L} \mathrm{NaCl}$ and $6 \mu \mathrm{g} / \mathrm{mL} \mathrm{AO})$. Within $3-5 \mathrm{~min}$ after the addition of $\mathrm{AO}$, the samples were analysed using a flow cytometer (FACSVerse, BD Biosciences). For each sample, a minimum of 10,000 events were analysed at a speed of 200 cells/s. Forward scatter (FSC), side scatter (SSC), green fluorescence (FL1) and red fluorescence (FL3) were collected after excitation with a blue laser $(488 \mathrm{~nm})$. The DNA fragmentation index (\%DFI: ratio of the percentage of cells with denatured, single-stranded DNA to total cells acquired (both with stable double-stranded DNA and denatured single-stranded DNA)) was calculated for each sample using FCS Express version 5 (Denovo Software, Thornhill, ON, Canada). 


\section{Protamine deficiency assay}

Sperm DNA compaction was assessed using chromomycin A3 (CMA3; Sigma-Aldrich), which competes with protamines for chromatin binding and provides an indirect measure of assessing protamines in spermatozoa, as per the method given by Zubkova and coworkers (Zubkova et al. 2005) with modifications. Frozen-thawed spermatozoa diluted in TNE buffer ( 2 million/mL) were washed with PBS by centrifugation (300g; $10 \mathrm{~min})$. To the washed spermatozoa, $100 \mu \mathrm{L} \mathrm{CMA3}$ staining solution was added, prepared by dissolving CMA3 in Mcllvaine's buffer $(17 \mathrm{~mL} 0.1 \mathrm{~mol} / \mathrm{L}$ citric acid mixed with $83 \mathrm{~mL} 0.2 \mathrm{~mol} / \mathrm{L} \mathrm{Na}_{2} \mathrm{HPO}_{4}$ and $10 \mathrm{mmol} / \mathrm{L} \mathrm{MgCl}_{2}, \mathrm{pH} 7.0$ ) to a concentration of $0.25 \mathrm{mg} / \mathrm{mL}$. The spermatozoa were incubated with CMA3 staining solution for $20 \mathrm{~min}$ in the dark. During this time, CMA3 bound to the protamine-deficient areas in the sperm DNA. Spermatozoa were then washed in PBS by centrifugation $(300 \mathrm{~g} ; 10 \mathrm{~min}$ ) and diluted in $500 \mu \mathrm{L} \mathrm{PBS}$, and $4 \mu \mathrm{L}$ propidium iodide (Molecular Probes) was added before analysing in a flow cytometer (FACSVerse, BD Biosciences) equipped with a blue laser $(488 \mathrm{~nm})$. The sperm population was gated using FSC and SSC, with debris exclusion performed by gating on PI-positive events collected through a 586/42 bandpass filter. Then the fluorescence from CMA3 staining, excited by the $405 \mathrm{~nm}$ violet laser, was collected using a $528 / 45$ bandpass filter and evaluated. Although this excitation wavelength is not at the excitation maximum (Tavalaee et al. 2010, Fahti et al. 2011), sufficient fluorescence was obtained for evaluation, as shown by the contour plot of PI fluorescence versus CMA3 fluorescence detected by flow cytometry (Supplementary Fig. 1, see section on supplementary data given at the end of this article).

\section{Free thiols and disulphide concentration assay}

The status of free thiols in spermatozoa was determined using monobromobimane (mBBr; Molecular Probes), a thiol-reactive fluorescent probe. $\mathrm{mBBr}$ gains fluorescence as it reacts with thiols and shows a blue fluorescence only after the formation of thioether. Free thiols and disulphide concentrations were assessed as per the procedure given by Seligman and coworkers (Seligman et al. 1994) and Zubkova and coworkers (Zubkova et al. 2005) with minor modifications. Spermatozoa diluted in TNE buffer (2 million/ $\mathrm{mL}$ ) were divided into two parts of 1 million spermatozoa. Spermatozoa in one tube were incubated $\left(37^{\circ} \mathrm{C}\right.$ for $\left.10 \mathrm{~min}\right)$ with $1 \mathrm{mmol} / \mathrm{L}$ of 1,4-dithiothreitol (DTT) to reduce disulphides to free thiols, while DTT was not added to the other tube. After centrifugation $(300 \mathrm{~g} ; 10 \mathrm{~min}), 100 \mu \mathrm{L} \mathrm{mBBr}$ solution (final concentration $0.5 \mathrm{mmol}$ ) was added to the sperm pellet and the sample was incubated in the dark for $10 \mathrm{~min}$. During this time, $\mathrm{mBBr}$ reacted and bound to free thiols. Spermatozoa were then washed in PBS by centrifugation (300 $\mathrm{g} ; 10 \mathrm{~min})$ and analysed in a flow cytometer (FACSVerse, BD Biosciences) after excitation of $\mathrm{mBBr}$ by the $405 \mathrm{~nm}$ violet laser. Fluorescence of $\mathrm{mBBr}$ was collected using a 528/45 bandpass filter, the sperm population was gated using FSC and SSC, and at least 10,000 sperm events were evaluated. A histogram of $\mathrm{mBBr}$ fluorescence detected by flow cytometry is given as Supplementary Fig. 2.
Disulphide concentrations were calculated by subtracting free thiols ( $\mathrm{mBBr}$ fluorescence in non-DTT-treated sample) from total thiols ( $\mathrm{mBBr}$ fluorescence in DTT-treated sample) for the corresponding sample and then dividing the value by two.

\section{Statistical analysis}

All statistical analyses were performed using IBM SPSS statistical software V 20.0 (IBM). First, the data distribution was analysed for normality using the Shapiro-Wilks test; the motility and \%DFI were arcsine transformed. The protamine deficiency, free thiols and disulphide values were subjected to $\log (\log 10)$ transformation before analysis to reduce the effect of outliers and to scale the variables.

The semen quality parameters were subjected to PCA to group the parameters with similar trends (Agarwal et al. 2003). All semen quality parameters were sorted into four components and the data matrix obtained was rotated using the Varimax procedure with the Kaiser normalisation to identify the highest positive correlation. Variables with a positive and high loading factor were identified. After PCA, the effects of smoking and male factor on semen parameters were analysed using independent sample $t$-test. The differences in the semen quality parameters in different age groups, infertility factors (male, female and unknown), and HRG C633T SNP were analysed using one-way ANOVA. Relationships among different semen parameters were analysed using Pearson correlation analysis. A $P$ value of $\leq 0.05$ was considered to be statistically significant. Results are shown as mean \pm S.E.M.

\section{Results}

The PCA from the seminal parameters showed that four extracted components accounted for $67 \%$ of the total variance (Table 1). The first principal component was related to traditional semen quality assessment parameters (sperm concentration $/ \mathrm{mL}$, total sperm concentration and yield after preparation). The second component was related to sperm DNA integrity (\%DFI and disulphide) and this parameter accounted for $16 \%$ of the variance. The third component was related to

Table 1 Results of principal component analysis based on different semen parameters in men from infertile couples.

\begin{tabular}{lclc}
\hline $\begin{array}{l}\text { Principal } \\
\text { component }\end{array}$ & Variance $(\%)$ & Parameter & $\begin{array}{c}\text { Loading } \\
\text { factor }\end{array}$ \\
\hline Component 1 & 27 & Sperm concentration $/ \mathrm{mL}$ & 0.92 \\
& & $\begin{array}{l}\text { Total sperm concentration } \\
\text { Yield after preparation }\end{array}$ & 0.79 \\
& & \%DFI & 0.70 \\
Component 2 & 16 & ODFulphide & 0.75 \\
& & Disule & 0.46 \\
Component 3 & 13 & Ejaculate volume & 0.92 \\
Component 4 & 11 & Free thiols & 0.89 \\
& & Progressive motility & 0.45 \\
Total & 67 & &
\end{tabular}

The loading factor represents the highest association between a given seminal parameter and the corresponding principal component. 
Table 2 Influence of age on different seminal parameters and sperm DNA integrity in men from infertile couples.

\begin{tabular}{lccr}
\hline Parameter & Age up to $\mathbf{3 0 . 7 5}$ years $(n=15)$ & Age between $\mathbf{3 0 . 7 5}$ and 41.52 years $(n=85)$ & Age above 41.52 years $(n=19)$ \\
\hline Ejaculate volume $(\mathrm{mL})$ & $3.33 \pm 0.34$ & $2.85 \pm 0.14$ & $3.08 \pm 0.37$ \\
Sperm concentration (million/mL) & $51.67^{\mathrm{a}} \pm 9.56$ & $61.21^{\mathrm{ab}} \pm 5.06$ & $80.84^{\mathrm{b}} \pm 12.14$ \\
Total sperm (million) & $183.20 \pm 41.08$ & $167.75 \pm 16.13$ & $242.24 \pm 48.37$ \\
Progressive motility (\%) & $54.87 \pm 5.04$ & $56.77 \pm 1.96$ & $62.63 \pm 3.11$ \\
Yield after preparation (millions) & $11.63^{\mathrm{a}} \pm 3.09$ & $13.62^{\mathrm{ab}} \pm 1.92$ & $26.22^{\mathrm{b}} \pm 5.43$ \\
\%DFI & $2.47^{\mathrm{a}} \pm 0.36$ & $5.60^{\mathrm{b}} \pm 1.10$ & $5.25^{\mathrm{b}} \pm 1.92$ \\
Protamine deficiency (mFI) & $18,977.60 \pm 1761.23$ & $16,184.16 \pm 628.18$ & $16,617.53 \pm 1052.5$ \\
Free thiols (mFl) & $5122.00 \pm 289.32$ & $5840.69 \pm 200.28$ & $5049.32 \pm 382.77$ \\
Disulphide (mFI) & $10,105.97 \pm 929.96$ & $10,125.72 \pm 519.01$ & $9399.50 \pm 931.75$ \\
\hline
\end{tabular}

Means with different superscripts in a row differ significantly $(P<0.05)$. Mean $($ age $)=36.13$, S.D. $=0.49$. Mean +1 s.D. $=41.52$,

mean -1 S.D. $=30.75$.

$\%$ DFI, DNA fragmentation index; mFI, mean fluorescence intensity.

the ejaculate volume, while the fourth component was related again to sperm DNA integrity (free thiols) and progressive motility of spermatozoa.

\section{Influence of age on seminal parameters}

As per the age, men were grouped into three categories based on mean and S.D. Men with age more than mean +1 s.D. were classified into the higher age group, while men with age less than mean-1 S.D. were classified into the lower age group; the rest (mean \pm 1 S.D.) were classified in the medium age group. Sperm concentration $(\mathrm{million} / \mathrm{mL})$ was significantly $(P<0.05)$ higher in men in the higher age groups compared to that in the lower age group (Table 2). Similarly, the number of spermatozoa obtained after density gradient centrifugation was also significantly higher in men in higher age group compared to that in lower age group. In contrast, sperm \%DFI was significantly $(P<0.05)$ lower in the comparatively younger age group than that in the higher age group. Other parameters did not differ among men in the three age groups.

\section{Influence of tobacco usage on seminal parameters}

The influence of using tobacco on seminal parameters among men from infertile couples is given in Table 3 . All the men in the smoking group used more than 20 cigarettes per day; however the data on smoking years was not available for calculation of a smoking index. Although the sample size was smaller in the smokers category, we observed a clear and significant $(P<0.01)$ difference in terms of sperm \%DFI between smokers and non-smokers. In contrast, the concentration of disulphide was significantly $(P<0.01)$ higher in smokers compared to non-smokers. Protamine deficiency and free thiols did not differ between the groups. Again, a significant difference was observed in sperm concentration, total sperm and yield after density gradient centrifugation, with significantly $(P<0.05)$ higher values among nonsmokers compared to smokers. Unlike smoking, using oral tobacco did not affect the seminal parameters significantly except for free thiols, which were significantly $(P<0.05)$ higher in those using oral tobacco compared to non-users.

\section{Seminal parameters among male, female and unknown factors of infertility}

The differences in the seminal profile of the three categories: male, female and unknown infertility factors are given in Table 4. The total sperm concentration was significantly $(P<0.05)$ lower in male infertility factor compared to that in unknown infertility factors. Sperm \%DFI was significantly $(P<0.01)$ higher in male infertility factor compared to both female and unknown infertility factors. Free thiols were significantly $(P<0.01)$ higher in spermatozoa of couples with unknown

Table 3 Effect of tobacco on different ejaculate qualities and sperm DNA integrity.

\begin{tabular}{lcccc}
\hline Parameter & Non-smokers $(n=111)$ & Smokers $(n=8)$ & No oral tobacco $(n=97)$ & Oral tobacco $(n=22)$ \\
\hline Ejaculate volume $(\mathrm{mL})$ & $3.06 \pm 0.14$ & $2.75 \pm 0.37$ & $3.04 \pm 0.15$ & $3.05 \pm 0.33$ \\
Sperm concentration (million/mL) & $64.26 \pm 4.54$ & $38.13 \pm 8.79$ & $64.15 \pm 4.90$ & $55.27 \pm 8.85$ \\
Total sperm (million) & $185.33^{\mathrm{b}} \pm 15.71$ & $98.03^{\mathrm{a}} \pm 12.07$ & $182.99 \pm 17.32$ & $163.89 \pm 25.60$ \\
Progressive motility (\%) & $57.87 \pm 1.73$ & $48.50 \pm 5.47$ & $58.37 \pm 1.77$ & $52.27 \pm 4.46$ \\
Yield after preparation (millions) & $15.92^{\mathrm{b}} \pm 1.79$ & $5.76^{\mathrm{a}} \pm 2.01$ & $14.55 \pm 1.71$ & $18.39 \pm 5.33$ \\
\%DFl & $4.50^{\mathrm{b}} \pm 0.59$ & $14.02^{\mathrm{a}} \pm 9.32$ & $5.00 \pm 0.97$ & $5.87 \pm 1.81$ \\
Protamine deficiency (mFI) & $16,448.07 \pm 545.47$ & $18,794.00 \pm 1989.40$ & $16,876.51 \pm 593.80$ & $15,412.14 \pm 1114.46$ \\
Free thiols (mFI) & $5616.50 \pm 156.87$ & $5724.38 \pm 1050.23$ & $5429.73^{\mathrm{b}} \pm 171.98$ & $6479.18^{\mathrm{a}} \pm 381.31$ \\
Disulphide (mFl) & $9772.69^{\mathrm{b}} \pm 396.11$ & $13,266.25^{\mathrm{a}} \pm 2558.32$ & $9728.89 \pm 469.36$ & $11,236.23 \pm 780.16$ \\
\hline
\end{tabular}

Means with different superscripts in a row within smokers and within oral differ significantly $(P<0.05)$.

$\% \mathrm{DFI}, \mathrm{DNA}$ fragmentation index; $\mathrm{mFI}$, mean fluorescence intensity. 
Table 4 Seminal parameters and sperm DNA integrity in subjects with male factor, female factor and unknown aetiology of infertility.

\begin{tabular}{lccr}
\hline & Male factor $(n=19)$ & Female factor $(n=28)$ & Unknown aetiology $(n=72)$ \\
\hline Ejaculate volume $(\mathrm{mL})$ & $2.68 \pm 0.31$ & $3.11 \pm 0.32$ & $3.11 \pm 0.16$ \\
Sperm concentration (million/mL) & $45.67 \pm 11.70$ & $59.57 \pm 6.35$ & $68.09 \pm 5.87$ \\
Total sperm (million) & $104.34^{\mathrm{a}} \pm 23.56$ & $155.36^{\mathrm{ab}} \pm 18.82$ & $208.66^{\mathrm{b}} \pm 21.93$ \\
Progressive motility (\%) & $51.68 \pm 5.59$ & $60.57 \pm 2.31$ & $57.42 \pm 2.13$ \\
Yield after preparation (millions) & $10.19 \pm 3.87$ & $13.94 \pm 2.88$ & $17.12 \pm 2.36$ \\
\%DFl & $12.05^{\mathrm{a}} \pm 2.46$ & $3.19^{\mathrm{b}} \pm 0.73$ & $4.17^{\mathrm{b}} \pm 1.13$ \\
Protamine deficiency (mFI) & $18,531.16 \pm 176.90$ & $16,908.21 \pm 1292.08$ & $15,980.08 \pm 634.59$ \\
Free thiols (mFI) & $4781.32^{\mathrm{a}} \pm 244.96$ & $5522.61^{\mathrm{ab}} \pm 375.68$ & $5885.39^{\mathrm{b}} \pm 205.75$ \\
Disulphide (mFI) & $9663.58 \pm 761.33$ & $10,171.00 \pm 1068.29$ & $10,034.76 \pm 506.08$ \\
\hline
\end{tabular}

Means with different superscripts in a row differ significantly $(P<0.05)$.

$\% \mathrm{DFI}$, DNA fragmentation index; $\mathrm{mFl}$, mean fluorescence intensity.

infertility factor. Other parameters did not differ in men among the three groups.

To understand the seminal parameters associated with each factor, PCA with groups was used. The data were grouped into male factor, female factor and unknown factor, and their distribution and tendency towards or away from specific sperm parameters were assessed. The relationship between the two principal components (principal component 1 along the $X$-axis and principal component 2 along the $\mathrm{Y}$-axis), the sperm parameters and the fertility factors is shown in Fig. 1. The groups with male, female or unknown infertility factors are indicated as red, blue and green ellipses. The size of each ellipse corresponds to the size (number of cases) of each corresponding group. The loading factors for each parameter in each respective principal component are shown in Table 1 . The loading factors indicate the correlation coefficients between the variables and factors. It is the \% of variance in that variable explained by the factor. This schematic representation (Fig. 1) shows the parameters defining each factor, distribution of the individual samples among the three factors of infertility and the predominant factor influencing the samples. There was a tendency for samples from men with male

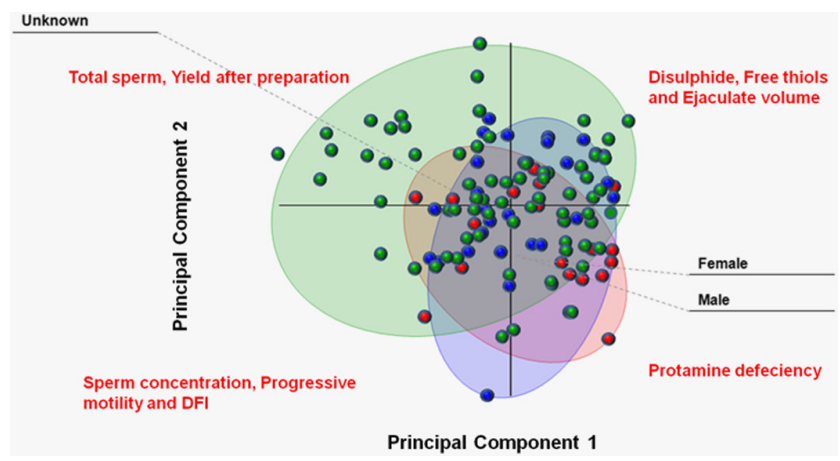

Figure 1 Figure shows the principal component 1 (in $\mathrm{X}$ axis) and principal component 2 (in $\mathrm{Y}$ axis) along with the distribution of samples among the three factors (red colour ellipse and dots indicate male factor; green colour ellipse and dots indicate female factor; and blue colour ellipse and dots indicate unknown factor). factor infertility to show lower sperm concentration and more DNA damage than samples from other males.

\section{Seminal parameters and ART outcome}

The seminal profile related to successful or unsuccessful outcomes (pregnancy confirmation) from ART (IVF and ICSI) is given in Table 5. No significant difference was observed between IVF success and IVF failure cases in any of the seminal parameters studied. Similarly, there was no significant difference in seminal parameters between ICSI success and ICSI failure cases. However, when the seminal parameters of men in IVF and ICSI group were compared, the men in IVF group had significantly $(P<0.05)$ higher sperm concentration, yield after preparation and significantly $(P<0.05)$ lower sperm \%DFI compared to the men in the ICSI group.

\section{Seminal parameters according to HRG C633T SNP}

The seminal parameters in men with HRG C633T SNP are given in Table 6. Although not enough to be statistically significant, the sperm concentration was lower in men with HRG 633T. Similarly, protamine deficiency was higher and disulphide concentration was lower in men with HRG 633T.

\section{Relationship among different seminal parameters in infertile men}

Having studied the influence of different factors on the seminal profile, we estimated the relationship among different seminal parameters using correlation analysis; the results are presented in Table 7 . Total sperm concentration was significantly positively correlated with ejaculate volume and sperm concentration. Progressive motility was significantly positively correlated with sperm concentration and total sperm count. Yield after density gradient centrifugation was significantly positively correlated with age, sperm concentration, total sperm count and progressive motility. Disulphide concentration was significantly negatively correlated with sperm concentration per millilitre and free thiols. 
Table 5 Seminal parameters and sperm DNA integrity in subjects with ART success or failure.

\begin{tabular}{lcccc}
\hline & IVF success $(n=31)$ & IVF failure $(n=41)$ & ICSI success $(n=11)$ & ICSI failure $(n=17)$ \\
\hline Ejaculate volume $(\mathrm{mL})$ & $2.81 \pm 0.26$ & $3.30 \pm 0.24$ & $2.55 \pm 0.34$ & $3.12 \pm 0.28$ \\
Sperm concentration (million/mL) & $77.32 \pm 9.52$ & $63.23 \pm 5.92$ & $35.70 \pm 13.39$ & $49.98 \pm 11.67$ \\
Total sperm (millions) & $199.45 \pm 31.29$ & $205.81 \pm 26.07$ & $108.27 \pm 41.09$ & $123.35 \pm 22.95$ \\
Progressive motility $(\%)$ & $58.00 \pm 2.90$ & $61.15 \pm 2.31$ & $54.55 \pm 8.14$ & $43.59 \pm 4.50$ \\
Yield after preparation (millions) & $16.96 \pm 3.52$ & $16.95 \pm 2.79$ & $5.67 \pm 2.79$ & $12.82 \pm 5.41$ \\
\%DFI & $3.30 \pm 0.94$ & $4.32 \pm 1.66$ & $11.45 \pm 3.95$ & $8.10 \pm 1.64$ \\
Protamine deficiency (mFI) & $16,469.19 \pm 1030.23$ & $15,854.23 \pm 783.85$ & $18,171.00 \pm 1758.73$ & $15,760.65 \pm 1280.20$ \\
Free thiols (mFI) & $5857.94 \pm 311.59$ & $5693.32 \pm 278.53$ & $4564.09 \pm 318.66$ & $5699.35 \pm 410.77$ \\
Disulphide (mFI) & $9032.97 \pm 866.59$ & $10,136.62 \pm 649.85$ & $9411.09 \pm 1147.86$ & $11,333.35 \pm 1102.69$ \\
\hline
\end{tabular}

$\% \mathrm{DFI}, \mathrm{DNA}$ fragmentation index; $\mathrm{mFI}$, mean fluorescence intensity.

The \%DFI was negatively correlated with free thiols in spermatozoa.

\section{Discussion}

While the incidence of infertility is increasing, with $4 \%$ more couples seeking ART per year, the improvement in ART outcome has been only marginal in spite of the efforts over a period of three decades (De Mouzon et al. 2010). One major reason for obtaining less than the anticipated success rates in ART is poor understanding of the causes of male infertility (Lewis 2013). In spite of the fact that DNA fragmentation is an important factor in male infertility, it is still under-evaluated and its inclusion in routine semen analysis is debated (Lewis 2013, Wright et al. 2014). In the present study, we analysed different seminal parameters in men from infertile couples and used PCA to identify the most relevant parameters that influence the fertility factor. We evaluated sperm DNA integrity using three fluorescent probes and established the relationship of sperm DNA integrity with seminal parameters, HRC C633T SNP and ART outcome.

In the present study, among the different factors of infertility, $16 \%$ of cases were attributed to male factor, $23.5 \%$ to female factor and the remainder to unknown factors. It was interesting to observe significantly higher $\%$ DFI in male factor infertility, indicating that sperm DNA integrity was compromised in these cases. Earlier studies have also reported significantly higher sperm DNA damage in male factor infertility compared to normal men (Høst et al. 2000, Saleh et al. 2002, Aydos et al. 2015), highlighting the significance of sperm DNA integrity as an important factor in male fertility. Another interesting finding was that the concentration of free thiols, an indicator of less highly condensed chromatin, was significantly higher in the unknown factor infertility group compared to the male factor infertility group. It has been reported that approximately $15 \%$ of infertile men have a normal spermiogram (Guzick et al. 1998); our results indicate that estimation of DNA integrity in these cases may help in identifying male infertility, which otherwise may not be possible with routine semen evaluation. Conventional semen analysis focuses on sperm concentration, viability, motility and morphology, and is a poor indicator of reproductive potential and pregnancy outcome (Shamsi et al. 2011). The PCA also supports our assumptions because $27 \%$ of the variations in the data were explained by the three parameters that assess sperm DNA integrity (\%DFI, free thiols and disulphide). Furthermore, the samples score figure of PCA and cluster analysis indicates that the male factor samples tend to aggregate towards the factors related to sperm DNA. Collectively, our findings indicate that although conventional semen evaluation parameters accounted for higher variability $(27 \%)$ in PCA, the three factors related to sperm DNA also accounted equally $(27 \%)$ to the variability, indicating that the assessment of sperm DNA may provide additional information about male infertility, especially in those with a normal spermiogram.

The assessment of sperm DNA damage in patients opting for ART is important because these techniques

Table 6 Seminal parameters and sperm DNA integrity in men according to HRG C633T SNP.

\begin{tabular}{lccc}
\hline & HRG 633T $(n=16)$ & HRG C633T $(n=40)$ & HRG C633 $(n=63)$ \\
\hline Ejaculate volume $(\mathrm{mL})$ & $3.00 \pm 0.35$ & $3.08 \pm 0.24$ & $3.03 \pm 0.18$ \\
Sperm concentration (million/mL) & $52.27 \pm 12.14$ & $62.55 \pm 7.86$ & $65.24 \pm 5.81$ \\
Total sperm (million) & $121.44 \pm 29.41$ & $183.05 \pm 24.73$ & $192.12 \pm 22.06$ \\
Progressive motility (\%) & $55.07 \pm 4.77$ & $53.80 \pm 3.26$ & $59.89 \pm 2.06$ \\
Yield after preparation (millions) & $12.93 \pm 5.19$ & $11.98 \pm 2.25$ & $17.99 \pm 2.59$ \\
\%DFl & $4.49 \pm 1.09$ & $6.21 \pm 2.20$ & $4.67 \pm 0.75$ \\
Protamine deficiency (mFI) & $18,451.13 \pm 420.54$ & $17,515.23 \pm 1020.96$ & $15,584.71 \pm 658.92$ \\
Free thiols (mFI) & $5335.60 \pm 609.21$ & $6096.95 \pm 272.38$ & $5351.51 \pm 194.20$ \\
Disulphide (mFI) & $9410.07 \pm 870.96$ & $9642.58 \pm 633.52$ & $10,198.81 \pm 607.68$ \\
\hline
\end{tabular}

$\% \mathrm{DFI}$, DNA fragmentation index; $\mathrm{mFI}$, mean fluorescence intensity; SNP, single nucleotide polymorphism. 


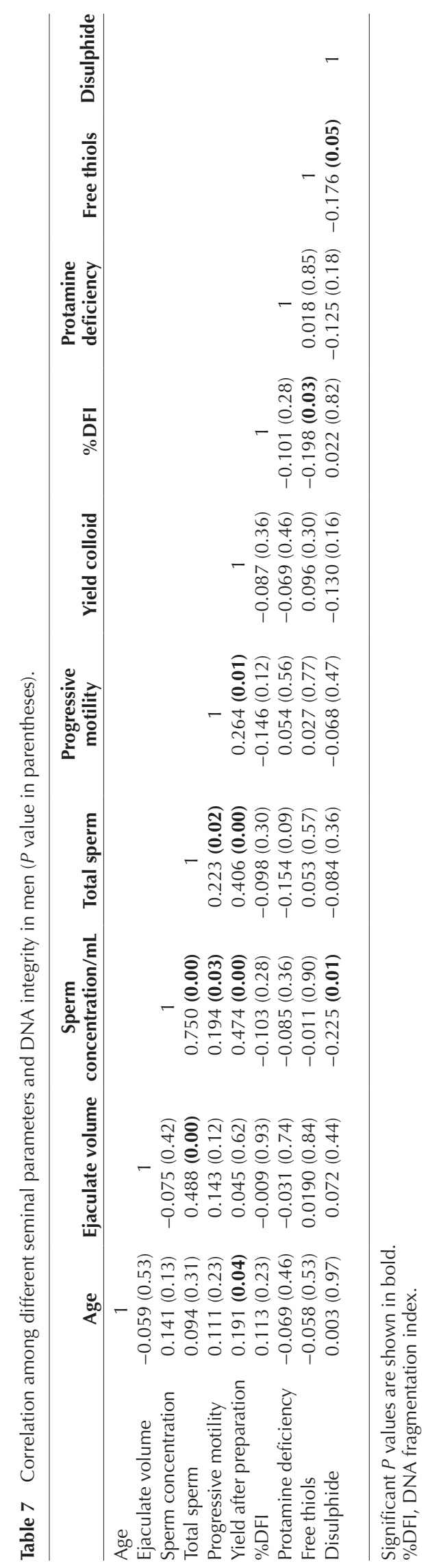

bypass the natural selection barriers of the female reproductive tract. When sperm with high DNA damage are used, the possibilities of transmitting genetic aberrations to the embryo/foetus are high (Zini 2011). The oocyte possesses DNA-repair mechanisms, unlike sperm, which allows them to deal with a certain amount of damage in the male component after fertilisation. However, a large genetic insult is believed to cause a severe disruption during embryonic development leading to loss of the embryo and failure of pregnancy (Collins \& Jones 2016).

Although the importance of intact sperm DNA is well recognised, sperm DNA integrity as a tool for predicting ART outcome has been the subject of debate. As indicated earlier, while some reports indicate no significant correlation between sperm DNA integrity and ART success (Morris et al. 2002, Gandini et al. 2004, Li et al. 2006), others report a significant reduction of pregnancy rates following ART among couples with high sperm DNA fragmentation (Larson et al. 2000, Benchaib et al.2003, Virro et al. 2004, Simon et al. 2010). In the present study, we did not observe a significant relationship between sperm DNA integrity and ART outcome, probably because the sperm samples had already been selected for intact DNA using the density gradient. Generally, a threshold value of $20 \%$ sperm DNA fragmentation with TUNEL assay has been suggested to distinguish between fertile controls and infertile men (Sergerie et al. 2005), and DNA fragmentation beyond this threshold level has been shown to negatively affect the ART outcome. In a recent report, it was shown that $80 \%$ of couples diagnosed with idiopathic infertility had sperm DNA damage and $40 \%$ of these couples had very high sperm DNA damage ( $>50 \%)$; the success of IVF was considerably lower for these couples (Lewis 2013). With the gradient-selected sperm samples in our study, the sperm DNA fragmentation was far lower than the threshold level, which might be the reason for not observing significant differences in \%DFI between IVF/ICSI success and failure outcomes. It would have been interesting to analyse the raw semen as well as the gradient-selected samples, but such samples were not available for us. Furthermore, the comparatively small sample size in the present study might also account for less variation in the semen parameters between ART success and failure outcomes.

The samples in the present study were not cryopreserved in what would normally be considered an optimal manner for preserving sperm function i.e. they were not frozen in the presence of cryoprotectant. At the time of sampling, the possibility of measuring DNA integrity had not been considered. However, earlier studies conducted on quantification of DNA damage in human spermatozoa also employed samples that were frozen without cryoprotectant. Sperm DNA quality (damage) was not altered by the process of freezing (Isachenko et al. 2004, Gianaroli et al. 2012). Based on 
these findings we do not consider that the method of preserving the samples would have affected the results, especially since all comparisons are made with samples treated in the same manner.

Except for the sperm concentration, no seminal parameters assessed in the present study had a significant relationship with sperm DNA integrity. However, the three methods used for the assessment of sperm DNA integrity were correlated with each other. Free thiol was negatively related to disulphide and \%DFI. Protamine deficiency did not show a significant correlation with any of the other parameters studied. Earlier studies performed to investigate the relationship between sperm DNA damage and conventional seminal parameters indicated an inverse correlation of DNA damage with sperm count, motility and morphology (Irvine et al. 2000, Evgeni et al. 2015). Several studies indicated that sperm from patients with abnormal sperm concentration, motility and morphology had high sperm DNA damage. A majority of these studies compared these parameters between fertile and infertile males and estimated the relationship. In the present study, we studied the relationship of these tests only in men from couples attending the fertility clinic. Furthermore, standard seminal parameters such as sperm concentration, motility and morphology can be highly variable over time within individuals (Alvarez et al. 2003), while measures of sperm DNA damage were shown to have good stability over time within individuals (Sergerie et al. 2005). In the present study, we used only one ejaculate per male and the possible implication of within-individual variations in seminal parameters on the relationship with sperm DNA cannot be ruled out. Nevertheless, our findings indicate that free thiol, disulphide and \%DFI had significant correlations and contribute to the sperm DNA integrity.

Although the cause of sperm DNA fragmentation is still unclear, it is widely accepted that sperm quality and infertility are associated with chromatin remodelling defects and oxidative stress. It is estimated that more than $10 \%$ of spermatogenesis impairment is due to genetic defects (Ferlin \& Foresta 2014, Krausz et al. 2015) and there is a growing literature on the genetic reasons for male infertility. It is likely that a considerable proportion of men among the infertile couples with no apparent male factor or female factor infertility might be affected with genomic male factor infertility including sperm DNA damage. Several studies indicated the involvement of genetic polymorphisms in specific genes in the aetiology of male infertility (Iguchi et al. 2006, Ji et al. 2012, Jiang et al. 2015). Recently it has been reported that male homozygous HRG 633T SNP carriers had low sperm count, sperm concentration and motility (Lindgren et al. 2016). In the present study, we investigated the effect of HRG C633T SNP on sperm DNA integrity. We could not find any significant relationship between HRG C633T SNP and sperm DNA integrity; however, protamine deficiency was higher and disulphide concentration was lower in men with HRG 633T. Other factors such as age and use of tobacco were also studied in relation to sperm DNA integrity. Our findings indicated an inverse relationship with age and sperm \%DFI. Older men had significantly higher sperm \%DFI compared to younger men, which is in agreement with other reports. Older men had increased sperm DNA damage (Schmid et al. 2007), whereas Singh and coworkers (Singh et al. 2003) observed that the percentage of highly damaged DNA sperm in the age group 36-57 years was significantly higher compared to the age group 20-35 years. Increasing paternal age has been shown to have negative effects on sperm quality, testicular function, epigenetic changes and DNA mutations leading to high levels of DNA fragmentation (Sharma et al. 2015). Although the sample size is small, we observed that smoking had a significant effect on sperm DNA fragmentation. It has been suggested that cigarette smoking negatively affects the reproductive process in males and spermatozoa from smokers have reduced fertilizing capacity (Ramlau Hansen et al. 2008). An increased proportion of spermatozoa with fragmented DNA in smokers compared with non-smokers have been reported by several researchers. Tobacco smoking induces double-stranded DNA breaks in sperm DNA in vitro (Arabi 2004). Interestingly, we observed that using oral tobacco had a positive and significant relationship with free thiols. Although available data do not conclusively demonstrate that smoking decreases male fertility, it is regarded as an infertility risk factor with altered semen qualities (Vine 1996, Mostafa 2010); our results suggest a negative effect of smoking on sperm DNA integrity.

\section{Conclusion}

Taken together, our findings indicate that density gradient-selected sperm samples still contained varying proportions of spermatozoa with DNA damage. Among the men from infertile couples, conventional semen analysis accounted for $27 \%$ of the variation in the population, whereas sperm DNA integrity also accounted for a similar proportion of the variation. Men classified as having unknown infertility based on the conventional semen analysis had higher sperm DNA damage than men from the female infertility factor category. Age and use of tobacco had a negative effect, while HRG C633T SNP had no significant effect on sperm DNA integrity, although tendencies indicate a need for larger studies. Male factor infertility is associated with parameters that indicate sperm DNA damage; thus the assessment of sperm DNA damage in addition to the conventional semen analysis may provide additional useful information about the type of male infertility especially in patients with a normal spermiogram. Future studies comparing the sperm DNA 
integrity in density gradient-selected and unselected spermatozoa from same subjects are needed to fully understand the role of sperm DNA integrity in male infertility and ART outcome.

\section{Supplementary data}

This is linked to the online version of the paper at http://dx.doi. org/10.1530/REP-17-0058.

\section{Declaration of interest}

J $M M$ is a shareholder of the company that manufactures the density gradient used in this study. However, the samples had already been prepared and stored prior to her involvement.

\section{Funding}

The first author acknowledges the Indian Council of Agricultural Research (ICAR) and National Dairy Research Institute, India for permitting him to undergo research cum training programme at the Department of Clinical Sciences/ Division of Reproduction, Swedish University of Agricultural Sciences, Uppsala, Sweden. The training was a part of 'Lal Bahadur Shastri Young Scientist Award' awarded by the ICAR to the first author.

\section{Acknowledgements}

The authors thank Maria Celina Abraham for technical assistance with the SCSA analysis. The authors would also like to thank Julius Hreinsson, researcher at the Department of Immunology, Genetics and Pathology, Uppsala University for his kind practical and assistance with this study. Additionally, the authors thank all the study participants for their generosity as well as all contributing staff at Fertilitetscentrum Stockholm, Sweden.

\section{References}

Agarwal A, Sharma RK \& Nelson DR 2003 New semen quality scores developed by principal component analysis of semen characteristics. Journal of Andrology 24 343-352. (doi:10.1002/j.1939-4640.2003. tb02681.x)

Alvarez C, Castilla JA, Martínez L, Ramírez JP, Vergara F \& Gaforio JJ 2003 Biological variation of seminal parameters in healthy subjects. Human Reproduction 18 2082-2088. (doi:10.1093/humrep/deg430)

Alpha Scientists in Reproductive Medicine \& ESHRE Special Interest Group of Embryology 2011 The Istanbul consensus workshop on embryo assessment: proceedings of an expert meeting. Human Reproduction 26 1270-1283. (doi:10.1093/humrep/der037)

Arabi M 2004 Nicotinic infertility: assessing DNA and plasma membrane integrity of human spermatozoa. Andrologia 36 305-310. (doi:10.1111/ j.1439-0272.2004.00623.x)

Aydos OS, Yükselten Y, Kaplan F, Sunguroğlu A \& Aydos K 2015 Analysis of the correlation between sperm DNA integrity and conventional semen parameters in infertile men. Turkish Journal of Urology 41 191-197. (doi:10.5152/tud.2015.98475)
Balhorn R, Reed S \& Tanphaichitr N 1988 Aberrant protamine 1/protamine 2 ratios in sperm of infertile human males. Experientia 44 52-55. (doi:10.1007/BF01960243)

Belokopytova IA, Kostyleva EI, Tomilin AN \& Vorob'ev VI 1993 Human male infertility may be due to a decrease of the protamine P2 content in sperm chromatin. Molecular Reproduction and Development 34 53-57. (doi:10.1002/mrd.1080340109)

Benchaib M, Braun V, Lornage J, Hadj S, Salle B, Lejeune H \& Guérin JF 2003 Sperm DNA fragmentation decreases the pregnancy rate in an assisted reproductive technique. Human Reproduction 18 1023-1028. (doi:10.1093/humrep/deg228)

Blank M \& Shoenfeld Y 2008 Histidine-rich glycoprotein modulation of immune/autoimmune, vascular, and coagulation systems. Clinical Reviews in Allergy and Immunology 34 307-312. (doi:10.1007/s12016007-8058-6)

Brandes M, Hamilton CJ, de Bruin JP, Nelen WL \& Kremer JA 2010 The relative contribution of IVF to the total ongoing pregnancy rate in a subfertile cohort. Human Reproduction 25 118-126. (doi:10.1093/ humrep/dep341)

Collins JK \& Jones KT 2016 DNA damage responses in mammalian oocytes. Reproduction 152 R15-R22. (doi:10.1530/rep-16-0069)

de Kretser DM 1997 Male infertility. Lancet 349 787-790. (doi:10.1016/ s0140-6736(96)08341-9)

de Mouzon J, Goossens V, Bhattacharya S, Castilla JA, Ferraretti AP, Korsak V, Kupka M, Nygren KG \& Andersen N 2010 Assisted reproductive technology in Europe, 2006: results generated from European registers by ESHRE. Human Reproduction 25 1851-1862. (doi:10.1093/humrep/ deq124)

Drasin T \& Sahud M 1996 Blood-type and age affect human plasma levels of histidine-rich glycoprotein in a large population. Thrombosis Research 84 179-188. (doi:10.1016/0049-3848(96)00174-0)

Erenpreiss J, Elzanaty S \& Giwercman A 2008 Sperm DNA damage in men from infertile couples. Asian Journal of Andrology 10 786-790. (doi:10.1111/j.1745-7262.2008.00417.x)

Esteves SC, Hamada A, Kondray V, Pitchika A \& Agarwal A 2012 What every gynecologist should know about male infertility: an update. Archives of Gynecology and Obstetrics 286 217-229. (doi:10.1007/ s00404-012-2274-x)

Evenson D \& Jost L 2000 Sperm chromatin structure assay is useful for fertility assessment. Methods in Cell Science 22 169-189. (doi:10.1023/ A:1009844109023)

Evenson DP \& Wixon RL 2005 Environmental toxicants cause sperm DNA fragmentation as detected by the Sperm Chromatin Structure Assay (SCSA). Toxicology and Applied Pharmacology 207 532-537. (doi:10.1016/j.taap.2005.03.021)

Evenson DP, Jost LK, Marshall D, Zinaman MJ, Clegg E, Purvis K, de Angelis P \& Claussen OP 1999 Utility of the sperm chromatin structure assay as a diagnostic and prognostic tool in the human fertility clinic. Human Reproduction 14 1039-1049. (doi:10.1093/humrep/14.4.1039)

Evgeni E, Lymberopoulos G, Gazouli M \& Asimakopoulos B 2015 Conventional semen parameters and DNA fragmentation in relation to fertility status in a Greek population. European Journal of Obstetrics and Gynecology and Reproductive Biology 188 17-23. (doi:10.1016/j. ejogrb.2015.02.026)

Fahti Z, Tavalaee M, Kiani A, Deemeh MR, Modaresi M \& Nasr-Esfahani MN 2011 Flow cytometry: a novel approach for indirect assessment of protamine deficiency by CMA3 staining, taking into account the presence of M540 or apoptotic bodies. International Journal of Fertility and Sterility 5 128-133.

Ferlin A \& Foresta C 2014 New genetic markers for male infertility. Current Opinion in Obstetrics and Gynecology 26 193-198. (doi:10.1097/ gco.0000000000000061)

Gandini L, Lombardo F, Paoli D, Caruso F, Eleuteri P, Leter G, Ciriminna R, Culasso F, Dondero F, Lenzi A et al. 2004 Full-term pregnancies achieved with ICSI despite high levels of sperm chromatin damage. Human Reproduction 19 1409-1417. (doi:10.1093/humrep/deh233)

Gianaroli L, Magli MC, Stanghellini I, Crippa A, Crivello AM, Pescatori ES \& Ferraretti AP 2012 DNA integrity is maintained after freeze-drying of human spermatozoa. Fertility and Sterility 97 1067-1073. (doi:10.1016/j. fertnstert.2012.02.014)

Gosálvez J, Gosálbez A, Arroyo F, Fernández JL \& López-Fernández C 2008 Assessing sperm DNA fragmentation in the field: an adaptation of 
sperm chromatin dispersion technology. Biotechnic and Histchemistry 83 247-252. (doi:10.1080/10520290802533528)

Guzick DS, Sullivan MW, Adamson GD, Cedars MI, Falk RJ, Peterson EP \& Steinkampf MP 1998 Efficacy of treatment for unexplained infertility. Fertility and Sterility 70 207-213. (doi:10.1016/S0015-0282(98)00177-0)

Hennis BC, Boomsma DI, Fijnvandraat K, Gevers Leuven JA, Peters M \& Kluft C 1995 Estrogens reduce plasma histidine-rich glycoprotein (HRG) levels in a dose-dependent way. Thrombosis and Haemostasis 73 484-487.

Høst E, Lindenberg S \& Smidt-Jensen S 2000 DNA strand breaks in human spermatozoa: correlation with fertilization in vitro in oligozoospermic men and in men with unexplained infertility. Acta Obstetricia et Gynecologica Scandinavica 79 189-193. (doi:10.1080/j.16000412.2000.079003189.x)

Iguchi N, Yang S, Lamb DJ \& Hecht NB 2006 An SNP in protamine 1: a possible genetic cause of male infertility? Journal of Medical Genetics 43 382-384. (doi:10.1136/jmg.2005.037168)

Irvine DS, Twigg JP, Gordon EL, Fulton N, Milne PA \& Aitken RJ 2000 DNA integrity in human spermatozoa: relationships with semen quality. Journal of Andrology 21 33-44.

Isachenko E, Isachenko V, Katkov II, Rahimi G, Schöndorf T, Mallmann P, Dessole S \& Nawroth F 2004 DNA integrity and motility of human spermatozoa after standard slow freezing versus cryoprotectant-free vitrification. Human Reproduction 19 932-939. (doi:10.1093/humrep/ deh194)

Jarow JP, Sharlip ID, Belker AM, Lipshultz LI, Sigman M \& Thomas AJ 2002 Best practice policies for male infertility. Journal of Urology 167 2138-2144. (doi:10.1016/S0022-5347(05)65109-9)

Ji G, Long Y, Zhou Y, Huang C, Gu A \& Wang X 2012 Common variants in mismatch repair genes associated with increased risk of sperm DNA damage and male infertility. BMC Medicine 17 10-49.

Jiang W, Sun H, Zhang J, Zhou Q, Wu Q, Li T, Zhang C, Li W, Zhang M \& Xia X 2015 Polymorphisms in protamine 1 and protamine 2 predict the risk of male infertility: a meta-analysis. Scientific Reports $\mathbf{5} 15300$. (doi:10.1038/srep15300)

Jones AL, Hulett MD \& Parish CR 2005 Histidine-rich glycoprotein: a novel adaptor protein in plasma that modulates the immune, vascular and coagulation systems. Immunology and Cell Biology 83 106-118. (doi:10.1111/j.1440-1711.2005.01320.x)

Kamath MS \& Bhattacharya S 2012 Demographics of infertility and management of unexplained infertility. Best Practice and Research Clinical Obstetrics and Gynaecology 26 729-738. (doi:10.1016/j. bpobgyn.2012.08.001)

Krausz C, Escamilla AR \& Chianese C 2015 Genetics of male infertility: from research to clinic. Reproduction 150 R159-R174. (doi:10.1530/ rep-15-0261)

Larson KL, DeJonge CJ, Barnes AM, Jost LK \& Evenson DP 2000 Sperm chromatin structure assay parameters as predictors of failed pregnancy following assisted reproductive techniques. Human Reproduction 15 1717-1722. (doi:10.1093/humrep/15.8.1717)

Lewis SEM 2013 The place of sperm DNA fragmentation testing in current day fertility management. Middle East Fertility Society Journal 18 78-82. (doi:10.1016/j.mefs.2013.01.010)

Li Z, Wang L, Cai J \& Huang H 2006 Correlation of sperm DNA damage with IVF and ICSI outcomes: a systematic review and meta-analysis. Journal of Assisted Reproduction and Genetics 23 367-376. (doi:10.1007/ s10815-006-9066-9)

Lindgren KE, Nordqvist S, Kårehed K, Sundström-Poromaa I \& Åkerud H 2016 The effect of a specific histidine-rich glycoprotein polymorphism on male infertility and semen parameters. Reproductive BioMedicine Online 33 180-188. (doi:10.1016/j.rbmo.2016.05.004)

Morris ID, Ilott S, Dixon L \& Brison DR 2002 The spectrum of DNA damage in human sperm assessed by single cell gel electrophoresis (Comet assay) and its relationship to fertilization and embryo development. Human Reproduction 17 990-998. (doi:10.1093/humrep/17.4.990)

Mostafa T 2010 Cigarette smoking and male infertility. Journal of Advanced Research 1 179-186. (doi:10.1016/j.jare.2010.05.002)

Nordqvist S, Karehed K, Stavreus-Evers A \& Akerud H 2011 Histidinerich glycoprotein polymorphism and pregnancy outcome: a pilot study. Reproductive BioMedicine Online 23 213-219. (doi:10.1016/j. rbmo.2011.04.004)
Nordqvist S, Kårehed K, Skoog Svanberg A, Menezes J \& Akerud H 2015 Ovarian response is affected by a specific histidine-rich glycoprotein polymorphism: a preliminary study. Reproductive BioMedicine Online 30 74-81. (doi:10.1016/j.rbmo.2014.09.016)

O'Flynn O'Brien KL, Varghese AC \& Agarwal A 2010 The genetic causes of male factor infertility: a review. Fertility and Sterility 93 1-12.

Oleszczuk K, Giwercman A \& Bungum M 2011 Intra-individual variation of the sperm chromatin structure assay DNA fragmentation index in men from infertile couples. Human Reproduction 26 3244-3248. (doi:10.1093/humrep/der328)

Patel KK, Poon IK, Talbo GH, Perugini MA, Taylor NL, Ralph TJ, Hoogenraad NJ \& Hulett MD 2013 New method for purifying histidinerich glycoprotein from human plasma redefines its functional properties. IUBMB Life 65 550-563. (doi:10.1002/iub.1168)

Ramlau Hansen CH, Thulstrup AM, Olsen J \& Bonde JP 2008 Parental subfecundity and risk of decreased semen quality in the male offspring: a follow-up study. American Journal of Epidemiology 167 1458-1464. (doi:10.1093/aje/kwn076)

Saleh RA, Agarwal A, Nelson DR, Nada EA, El-Tonsy MH, Alvarez JG, Thomas AJ Jr \& Sharma RK 2002 Increased sperm nuclear DNA damage in normozoospermic infertile men: a prospective study. Fertility and Sterility 78 313-318. (doi:10.1016/S0015-0282(02)03219-3)

Schmid TE, Eskenazi B, Baumgartner A, Marchetti F, Young S, Weldon R, Anderson D \& Wyrobek AJ 2007 The effects of male age on sperm DNA damage in healthy non-smokers. Human Reproduction 22 180-187. (doi:10.1093/humrep/del338)

Schulte RT, OhI DA, Sigman M \& Smith GD 2010 Sperm DNA damage in male infertility: etiologies, assays, and outcomes. Journal of Assisted Reproduction and Genetics 27 3-12. (doi:10.1007/s10815-009-9359-x)

Seligman J, Kosower NS, Weissenberg R \& Shalgi R 1994 Thiol-disulfide status of human sperm proteins. Journal of Reproduction and Fertility 101 435-443. (doi:10.1530/jrf.0.1010435)

Sergerie M, Laforest G, Bujan L, Bissonnette F \& Bleau G 2005 Sperm DNA fragmentation: threshold value in male fertility. Human Reproduction 20 3446-3451. (doi:10.1093/humrep/dei231)

Shamsi MB, Imam SN \& Dada R 2011 Sperm DNA integrity assays: diagnostic and prognostic challenges and implications in management of infertility. Journal of Assisted Reproduction and Genetics 28 1073-1085. (doi:10.1007/s10815-011-9631-8)

Sharma R, Agarwal A, Rohra VK, Assidi M, Abu-Elmagd M \& Turki RF 2015 Effects of increased paternal age on sperm quality, reproductive outcome and associated epigenetic risks to offspring. Reproductive Biology and Endocrinology 13 35. (doi:10.1186/s12958-015-0028-x)

Simon L, Brunborg G, Stevenson M, Lutton D \& McManus J 2010 Clinical significance of sperm DNA damage in assisted reproduction outcome. Human Reproduction 25 1594-1608. (doi:10.1093/ humrep/deq103)

Singh NP, Muller CH \& Berger RE 2003 Effects of age on DNA doublestrand breaks and apoptosis in human sperm. Fertility and Sterility $\mathbf{8 0}$ 1420-1430. (doi:10.1016/j.fertnstert.2003.04.002)

Tavalaee M, Kiani A, Arbabian M, Deemeh MR \& Esfahani MHN 2010 Flow cytometry: a new approach for indirect assessment of sperm protamine deficiency. International Journal of Fertility and Sterility 3 177-184.

Tocci A \& Lucchini C 2010 WHO reference values for human semen. Human Reproduction Update 16 559. (doi:10.1093/humupd/dmq019)

Vine MF 1996 Smoking and male reproduction: a review. International Journal of Andrology 19 323-337. (doi:10.1111/j.1365-2605.1996. tb00523.x)

Virro MR, Larson-Cook KL \& Evenson DP 2004 Sperm chromatin structure assay (SCSA) parameters are related to fertilization, blastocyst development, and ongoing pregnancy in in vitro fertilization and intracytoplasmic sperm injection cycles. Fertility and Sterility $\mathbf{8 1}$ 1289-1295. (doi:10.1016/j.fertnstert.2003.09.063)

Wang G, Guo Y, Zhou T, Shi X, Yu J, Yang Y, Wu Y, Wang J, Liu M, Chen X et al. 2013 In-depth proteomic analysis of the human sperm reveals complex protein compositions. Journal of Proteomics 79 114122. (doi:10.1016/j.jprot.2012.12.008)

Wright C, Milne S \& Leeson H 2014 Sperm DNA damage caused by oxidative stress: modifiable clinical, lifestyle and nutritional factors in male infertility. Reproductive BioMedicine Online 28 684-703. (doi:10.1016/j.rbmo.2014.02.004) 
Zini A 2011 Are sperm chromatin and DNA defects relevant in the clinic? Systems Biology in Reproductive Medicine 57 78-85. (doi:10.3109/193 96368.2010.515704)

Zini A, Bielecki R, Phang D \& Zenzes MT 2001a Correlations between two markers of sperm DNA integrity, DNA denaturation and DNA fragmentation, in fertile and infertile men. Fertility and Sterility 75 674-677. (doi:10.1016/S0015-0282(00)01796-9)

Zini A, Kamal KM \& Phang D 2001b Free thiols in human spermatozoa: correlation with sperm DNA integrity. Urology 58 80-84. (doi:10.1016/ S0090-4295(01)00997-9)
Zubkova EV, Wade M \& Robaire B 2005 Changes in spermatozoal chromatin packaging and susceptibility to oxidative challenge during aging. Fertility and Sterility 84 1191-1198. (doi:10.1016/j.fertnstert.2005.04.044)

Received 31 January 2017

First decision 28 February 2017

Revised manuscript received 17 March 2017

Accepted 29 March 2017 